\title{
A New Buck-Boost Converter for a Hybrid-Electric Drive Stand
}

P. Mašek

This paper describes work on the laboratory working stand for a hybrid-electric drive located in laboratory T2:H1-26.The basic idea is to operate the combustion engine in its optimal regime. In this regime the engine has the highest efficiency and minimal smoke exhaust. This optimal regime is only a small portion of its operation area. Because the engine has to operate in this area, it is necessary to add a new converter to the hybrid-electric stand. The new converter must be allowed to buck and boost and must operate as a current source in this regime.

Keywords: Buck-boost converter, hybrid-electric drive, power splitter, DC-DC converter, chopper.

\section{Status quo}

The stand consists of four electric machines, five convertors (rectifiers, inverters and choppers), a lot of various sensors (for measuring voltage, current, speed and torque) and another subcircuits and auxiliary circuits. A brief description its function is as follows. The main power source is the combustion engine. It is represented in our model as an electric machine.

This machine has a junction shaft with a special electric machine, the power splitter. This is a special synchronous machine, with a rotating rotor and stator. There are permanent magnets on the rotor, and there is winding on the stator. The transfer of mechanical energy is dependent on the current, that flows through the stator windings. The mechanical power is transferred to the power splitter, and there it is divided to two ways. The mechanical way is added to the power from the traction motor (the third machine), and this resulting power is transferred to the wheel of the car. The mechanical output of the power splitter has one junction shaft with a traction motor and the last electric machine. The fourth electric machine absorbs the mechanical power and represents the flow resistance of air, the rolling-resistance force and the rise and fall of the road. The electric energy path is from the power splitter's rectifier to the DC circuit. Another two converters are connected to this circuit. The first is the DC-DC convertor, which controls the charging and discharging of the supercapacitor. The second is the inverter, which controls the traction engine.

\section{Control of the stand}

The stand is very complicated at the present time. Many assistants are needed for the measurements, (at least four).

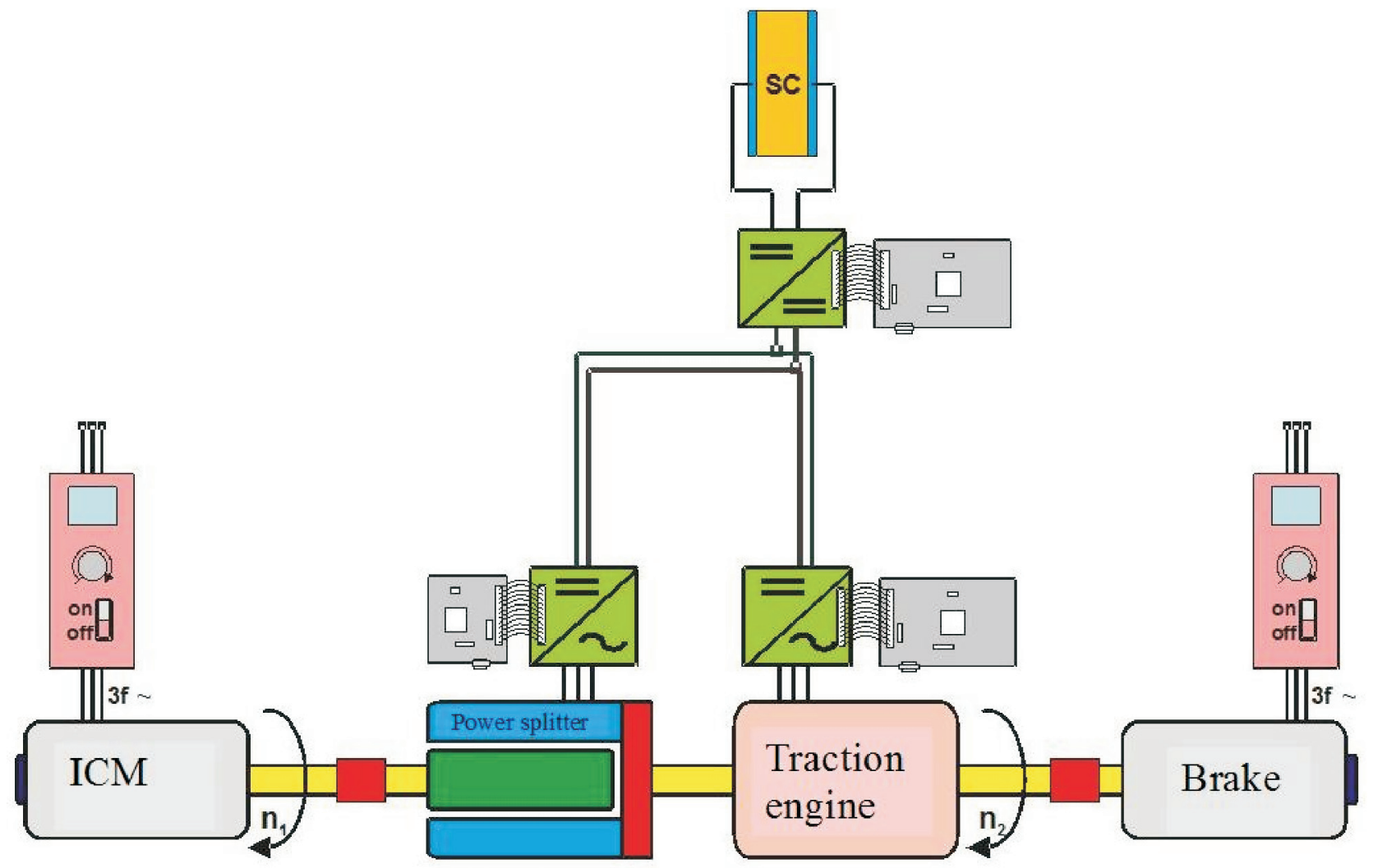

Fig. 1: Principal scheme of the hybrid-electric drive stand. The red block will be the new buck-boost converter 
Table 1: Electrical and mechanical parameters of the electrical engines

\begin{tabular}{|c|c|c|c|c|}
\hline & Power $(\mathrm{kW})$ & RPM & Voltage (V) & Nominal Current (A) \\
\hline ICM Brake & 7.5 & 2930 & D/Y 400/690 & D/Y 13.8/8 \\
\hline Power splitter & 5 & $\begin{array}{c}\text { different } 6000 \\
\text { one shaft } 4000\end{array}$ & 468 & \\
\hline Traction Engine & 3 & 1420 & D/Y 230/400 & D/Y 11.1/6.4 \\
\hline
\end{tabular}

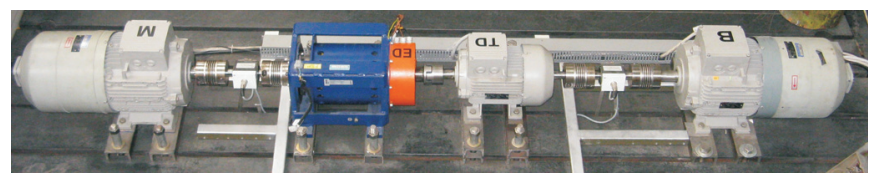

Fig. 2: True configuration of the hybrid-electric stand

Each converter has a different method of control, and only the local controlling is possible.

Each measuring instrument has a different method for reading the value and no remote reading for the measured value is available. We are now working very intensive to connect all converters and all sensors to one main computer. This computer has many $\mathrm{AD}$ converters and can read the value from the sensors, which have the current output. This computer has a great amount of binary output and output with the DA converters, which allows us to control the converters

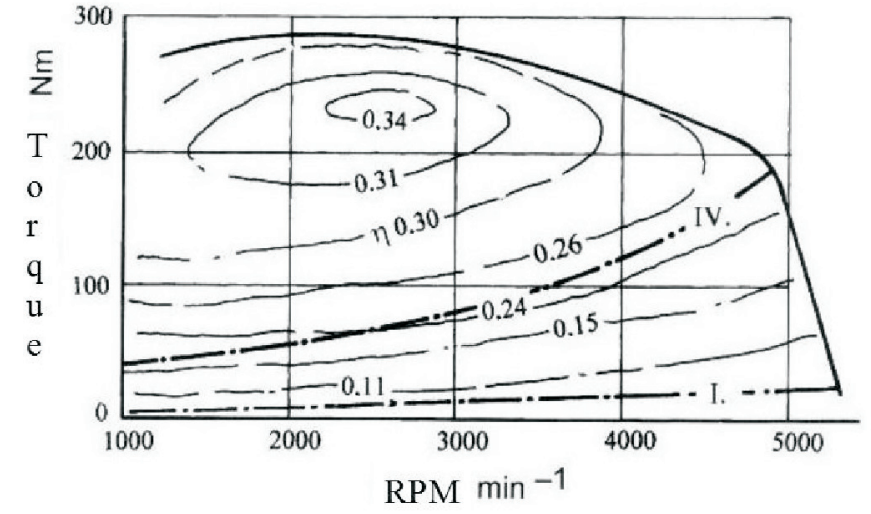

I - first, IV - fourth speed gear

Fig. 3: This graph shows the efficiency on the working space of the standard combustion engine

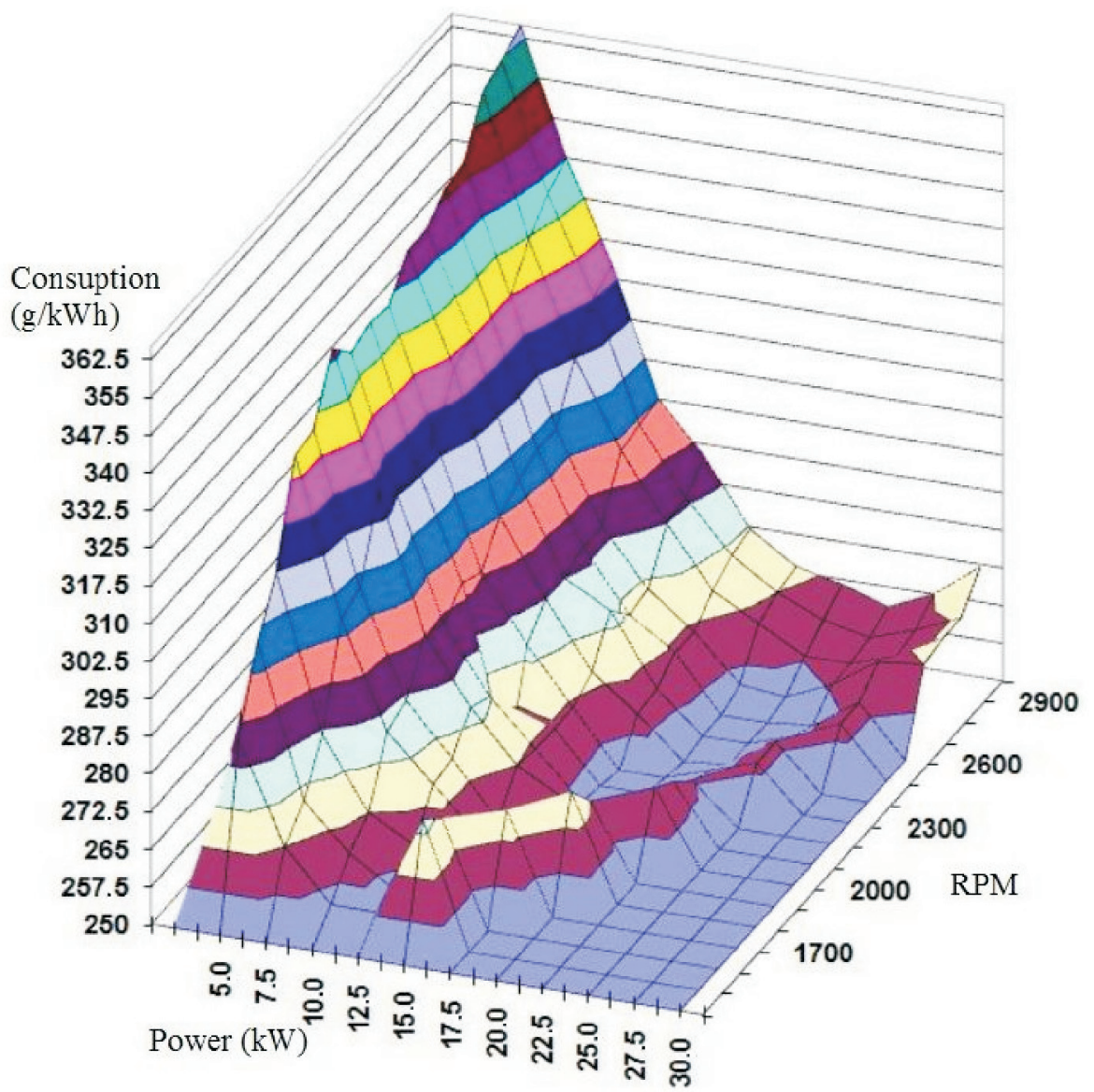

Fig. 4: This graph shows the fuel consumption of a car on the working space 
or switch on/off particular components or the whole working stand.

This computer can assemble the data from the whole workplace. From an analysis of this data it is possible to correct the input parameters.

This system enables the machinery to be tested in special driving cycles, for example in European driving cycles. After this simulation we can verify the data from the virtual calculation and simulation.

\section{The provision design}

Another task is being undertaken at the same time as the work on data acquisition. It is important to supplement the work stand with one component or one function. The measurements have detected a major problem of voltage instability in the DC circuit. The power from the power splitter is restricted and in one moment it is constant.

\subsection{Voltage instability}

However the power taken by the traction motor is very variable and its volume depends on the driver's requirement to accelerate or brake the car. Another contribution to the voltage instability in a DC circuit is the converter of the supercapacitor. its program tries to hold the voltage in a DC circuit. But its time constant is too small and there is no storage element in the DC circuit. There is a pulse process, and the voltage in the DC circuit rises and falls with time, depending on the speed of the regulation loop. It is only possible to remove this process by adding a storage element to the DC circuit. The storage element will stabilize the voltage in the DC circuit.

The optimal voltage is in the middle of the power splitters operating area i.e. $240 \mathrm{~V}$. The voltage from the power splitter depends on the differential speed. From the analysis of the power splitter operation it results that in about $50 \%$ of the working time the voltage is greater than $240 \mathrm{~V}$, and we must reduce the voltage. In about $50 \%$ of the working time the voltage is lower than $240 \mathrm{~V}$, and we must raise the voltage.

\subsection{Reasons for building the new converter}

The supply voltage is at present controlled by the special rectifier from the power splitter. The great disadvantage is that it needs greater voltage on its output than on its input.

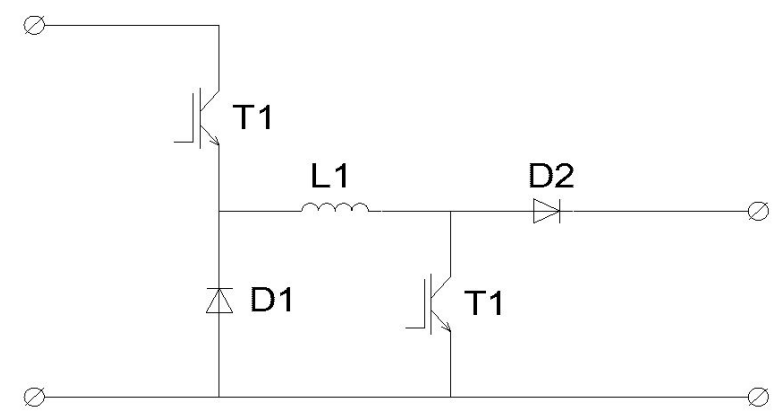

Fig. 6: An electrical scheme of the new buck-boost converter

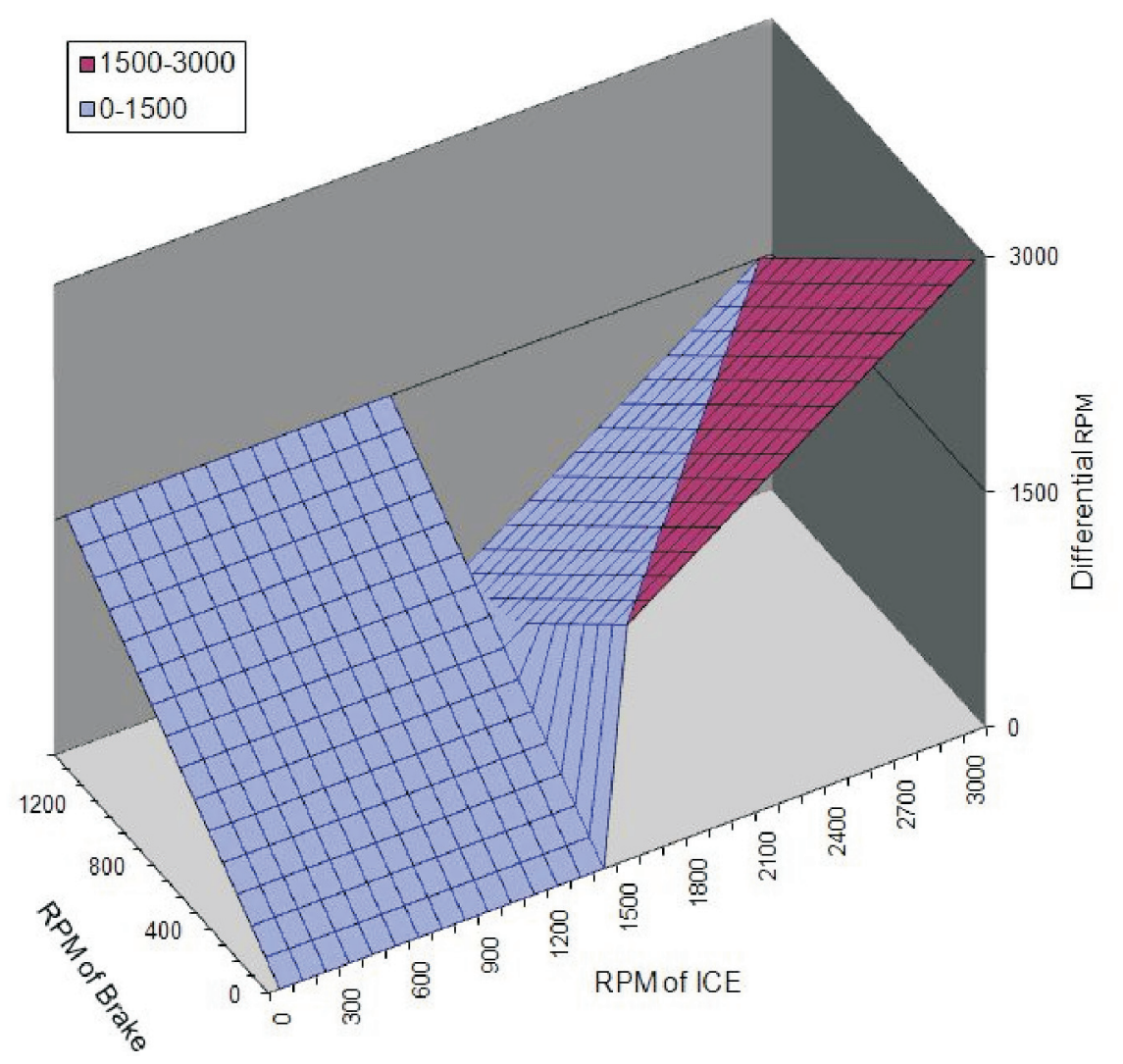

Fig. 5: This graph shows the differential RPM of the power splitter depending on the RPM of the combustion engine and the RPM of the brake. This graph takes into account the working area of the two engines. In the blue area it is necessary to boost and in the violet area it is necessary to buck.

Note: The RPM of ICE up to 1500 is 0 . The engine works between 1500 and 3000. 
Table 2: Anticipated specifications of all components from Fig. 6

\begin{tabular}{|l|l|}
\hline T1, T2 & $\begin{array}{l}\text { IGBT 1200V; 100A; } \\
\text { ref.: Eupec BSM100GB120DLCK }\end{array}$ \\
\hline D1, D2 & $\begin{array}{l}2 \times 600 \mathrm{~V} ; 2 \times 40 \mathrm{~A} ; \\
\text { ref.: Semic Trade HFA120EA60 }\end{array}$ \\
\hline L1 & inductor with a ferrite core $\sim 1 \mathrm{mH}$ \\
\hline Frequency & $5 \div 50 \mathrm{kHz}$ \\
\hline
\end{tabular}

When this condition is false, this rectifier functions as a normal 3-phase uncontrolled diode rectifier, so all advantages are out of order and we cannot control the rectifier. Because the existing regulation does not allow the demagnetization of the power splitter rotor, we cannot reduce the output voltage from the rectifier. To make a new program to control the rectifier to allow demagnetization of the permanent magnets on the power splitter rotor goes beyond range of my work. It was therefore decided that a new converter would be built. It will be a Buck-Boost pulse convertor, which will work as a controlled current source. It will work on the output from the power splitter's rectifier, and will function only as an uncontrolled rectifier.

\subsection{Contribution of the new converter}

The new converter brings two advantages. First, it simplifies the connection between the power splitter's rectifiers and the main control computer. An evolutionary kit with DSP 56F805 by Freescale (Motorola) is used to control the rectifier.

This evolutionary kit is a powerful unit. It is suitable as a self-contained unit. A problem is that this kit can be controlled only locally by four integrated switches or by volume-control connected to ADC. Remote control is possible only via special software by Freescale.

LabView is the program on the main computer, that is used for visualization. We have no simple way to connect these two systems. My idea is that the new convertor will be controlled by a programmable gate array by National Instruments, which is compatible with LabView.

A second advantage is that it is simpler to start making measurements. On the stand, incremental optical sensors are used as sensors for measuring speed. The control system of the power splitter's rectifiers needs information about the position of the rotor and stator. There are only incremental sensors, no absolute position sensors.

When we want to start making measurements, we first have to rotate both shafts at least one rotation. My idea is that

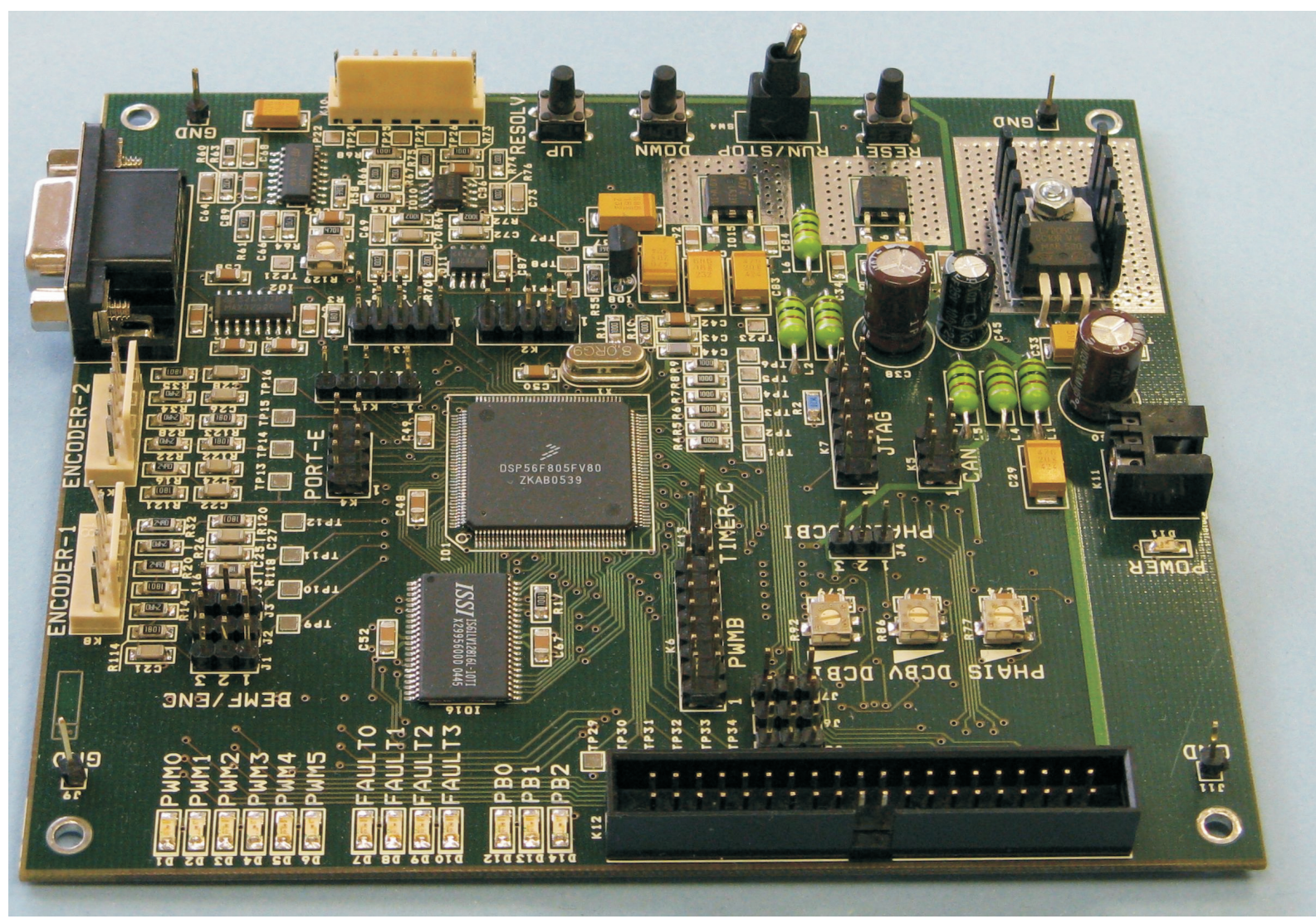

Fig. 7: Evolutionary kit with DSP 56F805

Main parameters:

- 16-bit $+3.3 \mathrm{~V}$ controller operating at $80 \mathrm{MHz}$

- 4-Channel 10-bit Serial D/A

- On-board Parallel JTAG Host Target Interface, with a connector for a PC printer port cable

- RS-232 interface for easy connection to a host processor

- CAN interface for high speed communications

- Primary and secondary UNI-3 Motor interface Encoder/Hall-Effect interface: Over-Voltage sensing; Over-Current sensing; Phase Current sensing; Back-EMF sensing; Temperature sensing; Zero Crossing detection; Pulse Width Modulation etc 


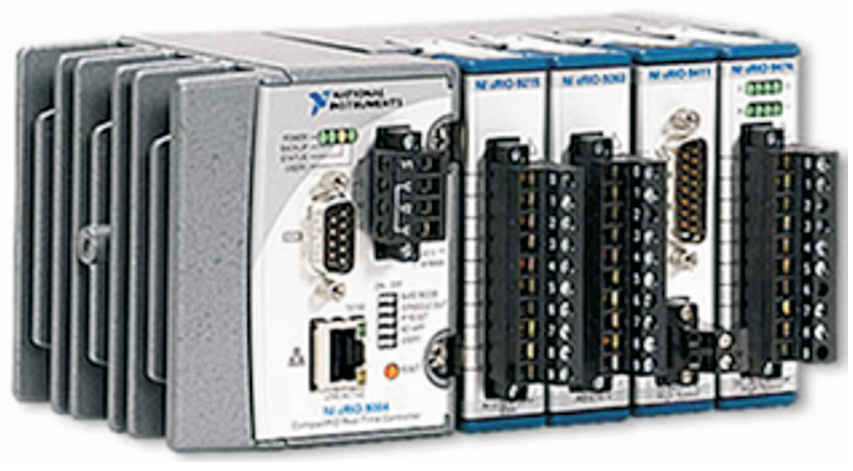

Fig. 8: CompactRio - this programmable automation controller is a low-cost reconfigurable control and acquisition system designed for applications that require high performance and reliability. The system combines an open embedded architecture with small size, extreme ruggedness, and hot-swappable industrial I/O modules. NI CompactRIO is powered by reconfigurable I/O (RIO) FPGA technology. NI CompactRio is compatible with the LabView development system.

the rectifier will work only as an uncontrolled diode rectifier, and then we will not need information about the position of the rotor and stator and we will not rotate the shafts first.

\section{Summary}

The new convertor will consist ofm two parts, the step-up part and the step-down part. The two parts will be operated separately. There will be one power transistor in each part. Both transistors will be controlled by a microcomputer, probably by a Compact Rio unit. The microcomputer will measure the input and output voltage and the transient current. The computer will decide from this data which part will work. The current regulation will hold the transient current constant, which means that the torque will be constant and the power carried to the mechanical way will also be constant.

The main idea of hybrid-electric engines is to build more efficiency cars, which will be fuel-saving and will be emissionless. I hope that my work will contribute to this field of research.

\section{Acknowledgements}

The research described in this paper was supervised by Prof. Ing. Zdeněk Čeřovský, DrSc., FEE CTU in Prague and supported by the Josef Božek Research Centre of Engine and Automotive Engineering.

\section{References}

[1] Cundev, D.: Driving Regime of the Recuperative System with Super-capacitor, Czech Technical University in Prague, Faculty of Electrical Engineering, Department of Electric Drives and Traction, 2008.

\section{Petr Mašek}

e-mail: masekp4@fel.cvut.cz

Department of Electric Drives and Traction

Czech Technical University in Prague

Faculty of Electrical Engineering

Technická 2

16627 Prague, Czech Republic

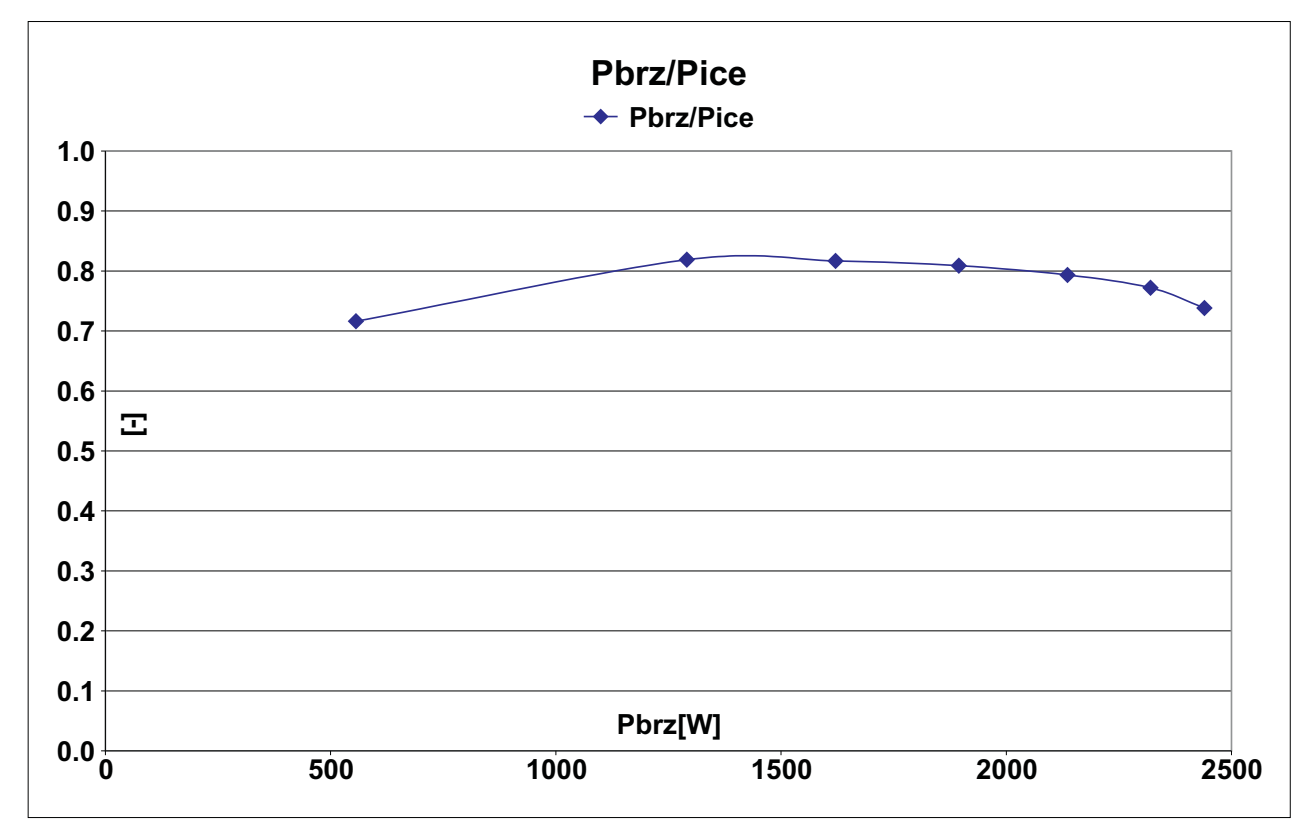

Fig. 9: A graph showing the efficiency of the energy transfer from an Internal Combustion Engine to a car wheel (Pbr) measured on a hybrid-electric stand. If the combustion engine works in its optimal area, the energy can be transferred to the wheel with high efficiency. 